15

\title{
Возвратные фазы в компенсированных феррохолестериках
}

\author{
(C) А.Н. Захлевных, К.В. Кузнецова \\ Пермский государственный национальный исследовательский университет, \\ Пермь, Россия \\ E-mail: anz@psu.ru
}

(Поступила в Редакцию 15 марта 2017 г.)

Теоретически изучено влияние магнитного поля на ориентационные и магнитные свойства компенсированного феррохолестерика - суспензии игольчатых феррочастиц в холестерическом жидком кристалле. Рассмотрен фазовый переход феррохолестерик-ферронематик в магнитном поле, перпендикулярном оси спиральной структуры. Исследована зависимость поля перехода в ферронематическую фазу от материальных параметров суспензии, а также шага спиральной структуры и намагниченности от напряженности поля. Показана возможность существования возвратной феррохолестерической фазы.

Работа выполнена при поддержке РФФИ (грант № 16-42-590539).

DOI: 10.21883/FTT.2017.09.44859.079

\section{1. Введение}

Холестерические жидкие кристаллы (ХЖК) обладают спиральной надмолекулярной структурой и вследствие этого замечательными оптическими свойствами [1]. Это обусловлено тем, что шаг спиральной структуры ХЖК обладает высокой чувствительностью к внешним воздействиям, в частности к электрическим и магнитным полям. Известно [1], что спиральная структура ХЖК с положительной анизотропией диамагнитной восприимчивости $\chi_{a}$ или диэлектрической проницаемости $\varepsilon_{a}$ раскручивается во внешнем магнитном или электрическом поле, приложенном перпендикулярно оси спирали. Вследствие малости $\chi_{a}$ этот эффект наблюдается лишь в сильных магнитных полях. Для повышения магнитной восприимчивости ХЖК можно внедрить в него игольчатые магнитные частицы. Такие магнитные суспензии, приготовленные на основе ХЖК, получили название феррохолестериков (ФХ) [2]. В них ввиду ориентационной связи между иглообразными магнитными частицами и ХЖК-матрицей вектор намагниченности спирально закручен в пространстве вокруг некоторой оси. В этом смысле ФХ представляет собой жидкокристаллический аналог геликоидальных ферро- или антиферромагнетиков. В отличие от твердых ферроили антиферромагнетиков магнитные частицы в ФХ имеют возможность пространственного перемещения, мигрируя в те области образца, где минимальна их магнитная и ориентационная энергия (так называемый эффект сегрегации [2]).

Наличие жидкокристаллической и магнитной подсистем в композитной системе - феррохолестерике приводит к тому, что ФХ обладает двумя механизмами ориентационного взаимодействия с магнитным полем: квадрупольным (диамагнитным) взаимодействием магнитного поля с жидкокристаллической (ЖК) матрицей и дипольным (ферромагнитным) влиянием поля на магнитные моменты частиц. Феррохолестерики привлекают внимание в связи с возможностью посредством слабых внешних магнитных полей изменять спиральность образуемой текстуры и тем самым легко модулировать спектральный состав отраженного света.

Выше температуры перехода в мезофазу такие суспензии изотропны и по свойствам близки к обычным магнитным жидкостям. При охлаждении в ЖК-матрице происходит фазовый переход в жидкокристаллическое состояние и возникает дальний ориентационный порядок. Если охлаждение осуществляется в отсутствие магнитного поля, то образующаяся суспензия получается компенсированной с нулевой макроскопической намагниченностью, так как в ней имеются равные доли феррочастиц с противоположно направленными магнитными моментами. Если охлаждение суспензии из изотропной фазы производится в подмагничивающем поле, то в упорядоченной фазе магнитные моменты частиц оказываются упорядоченными. Таким образом, в зависимости от способа приготовления различают намагниченные (ЖК-аналоги геликоидальных ферромагнетиков) и компенсированные (ЖК-аналоги геликоидальных антиферромагнетиков) феррохолестерики.

Первые синтезированные феррохолестерики [3] не отличались устойчивостью. Однако в последние годы в связи с успехами синтеза геликоидальных ферросуспензий появилось множество экспериментальных работ [4-13], в которых изучаются различные свойства этих новых мягких магнитных материалов. Имеющиеся теоретические работы посвящены в основном геликоидальным феррохолестерикам [14-20]; компенсированные ФХ до сих пор не рассматривались. В настоящей работе изучается влияние магнитного поля на ориентационную структуру компенсированного феррохолестерика с гомеотропным сцеплением магнитных частиц с ХЖК-матрицей; случай планарного сцепления исследован в недавней работе [21]. 


\section{2. Свободная энергия феррохолестерика}

Рассмотрим ориентационные и магнитные свойства компенсированного ФХ с жестким гомеотропным сцеплением между феррочастицами и молекулами ХЖК в магнитном поле, перпендикулярном оси спиральной структуры. Приложенное к нему магнитное поле воздействует как на магнитные моменты феррочастиц (дипольный механизм), так и на диамагнитную холестерическую матрицу (квадрупольный механизм). Будем полагать, что диамагнитная анизотропия матрицы $\chi_{a}$ положительна, тогда директор будет стремиться повернуться в направлении поля, а гомеотропные условия сцепления (магнитные частицы внедрены в ХЖК-матрицу таким образом, что их главные оси перпендикулярны локальному директору $\mathbf{n}$ ) приводят к тому, что эти механизмы являются конкурирующими, так как стремятся повернуть директор феррохолестерика в противоположных направлениях.

Вызванное магнитным полем искажение ориентационной структуры ФХ можно рассматривать в рамках континуальной теории, основанной на функционале свободной энергии $\mathscr{F}=\iiint F d V$. Здесь плотность свободной энергии $F$ имеет вид $[2,16,22,23]$

$$
F=F_{1}+F_{2}+F_{3}+F_{4},
$$

где

$$
\begin{gathered}
F_{1}=\frac{1}{2}\left[K_{11}(\operatorname{div} \mathbf{n})^{2}+K_{22}\left(\mathbf{n} \operatorname{rot} \mathbf{n}+q_{0}\right)^{2}+K_{33}(\mathbf{n} \times \operatorname{rot} \mathbf{n})^{2}\right], \\
F_{2}=-\frac{\chi_{a}}{2}(\mathbf{n} \mathbf{H})^{2}, \\
F_{3}=-M_{s}\left(f_{+}-f_{-}\right)(\mathbf{m H}), \\
F_{4}=\frac{k_{\mathrm{B}} T}{v}\left[f_{+} \ln f_{+}+f_{-} \ln f_{-}\right],
\end{gathered}
$$

$K_{11}, K_{22}, K_{33}-$ модули ориентационной упругости Франка, $q_{0}-$ собственное волновое число спиральной структуры ХЖК, $\chi_{a}=\chi_{\|}-\chi_{\perp}-$ анизотропия диамагнитной восприимчивости ХЖК, $M_{s}$ - намагниченность насыщения материала феррочастиц, $v$ - объем частицы, $k_{\mathrm{B}}$ - постоянная Больцмана, $T-$ температура, $\mathbf{m}$ - единичный вектор намагниченности, $\mathbf{n}$ - директор жидкого кристалла, $f_{+}$и $f_{-}$- объемные доли магнитных частиц в суспензии с магнитными моментами, направленными вдоль $\mathbf{m}$ и $-\mathbf{m}$ соответственно. Мы рассматриваем компенсированный $\Phi Х$, т. е. в отсутствие поля $f_{+}=f_{-}=f_{0} / 2$. Среднюю объемную долю частиц в суспензии полагаем малой $\left(f_{0}=N v / V \ll 1\right)$, поэтому магнитными диполь-дипольными взаимодействиями частиц можно пренебречь (здесь $N$ - число примесных частиц, $V$ - объем суспензии).

Слагаемое $F_{1}$ в выражении (1) представляет собой объемную плотность энергии ориентационно-упругих деформаций директора $\mathbf{n} ; F_{2}$ описывает взаимодействие диамагнитной ЖК-матрицы с магнитным полем $\mathbf{H}$; вклад $F_{3}$ отвечает взаимодействию магнитных частиц с полем; $F_{4}$ описывает вклад энтропии смешения идеального раствора частиц в свободную энергию.

Вследствие спиральной структуры ХЖК вектор намагниченности твердой фазы оказывается в отсутствие поля закручен в пространстве вокруг оси, перпендикулярной $\mathbf{n}$ и $\mathbf{m}$, формируя спираль ФХ с шагом $p_{0}=2 \pi / q_{0}$. Под действием внешнего магнитного поля $\mathbf{H}=(0, H, 0)$, перпендикулярного оси спирали $Ф \mathrm{X}$, ориентационная структура теряет геликоидальный характер, и зависимость компонент директора и намагниченности от координаты $z$ перестает быть гармонической:

$$
\begin{gathered}
\mathbf{n}=(\cos \varphi(z, H), \sin \varphi(z, H), 0), \\
\mathbf{m}=(-\sin \varphi(z, H), \cos \varphi(z, H), 0),
\end{gathered}
$$

где $\varphi(z, H)$ - угол поворота директора и намагниченности, отсчитываемый от оси $x$.

Подставим выражения (2) и (3) в плотность свободной энергии (1)

$$
\begin{aligned}
F & =\frac{K_{22}}{2}\left(\frac{d \varphi}{d z}-q_{0}\right)^{2}-\frac{\chi_{a} H^{2}}{2} \sin ^{2} \varphi \\
& -M_{s}\left(f_{+}-f_{-}\right) H \cos \varphi+\frac{k_{\mathrm{B}} T}{v}\left(f_{+} \ln f_{+}+f_{-} \ln f_{-}\right) .
\end{aligned}
$$

Для перехода к безразмерным переменным выберем в качестве единицы длины величину $q_{0}^{-1}$, тогда безразмерная координата $\tilde{z}=q_{0} z$. Введем обозначения для безразмерных параметров [14]

$$
\xi \equiv \frac{M_{s} f_{0}}{q_{0} \sqrt{K_{22} \chi_{a}}}, \quad \kappa \equiv \frac{k_{\mathrm{B}} T f_{0}}{v K_{22} q_{0}^{2}}, \quad h=\frac{H}{H_{q}} .
$$

Здесь $h$ представляет собой безразмерную напряженность поля, измеренную в единицах поля перехода в нематическую фазу $H_{q}=q_{0} \sqrt{K_{22} / \chi_{a}}$ в беспримесном ХЖК [1]. Параметр $\xi$ характеризует режимы раскручивания спирали (при $\xi \gg 1$ преобладает дипольный режим, при $\xi \ll 1$ - квадрупольный режим). Параметр $\kappa$ отвечает за эффект сегрегации [2]: под действием магнитного поля Н магнитные частицы мигрируют вдоль оси спирали в те ее области, в которых минимальна их магнитная и ориентационная энергия. При $\kappa \gg 1$ сегрегация частиц несущественна.

Заметим, что концентрации двух магнитных подсистем компенсированного ФХ удовлетворяют условию постоянства числа частиц в суспензии

$$
\int\left(f_{+}+f_{-}\right) d V=N v \text {. }
$$

Определим приведенные объемные доли $g_{ \pm}$частиц соотношением $f_{ \pm}=f_{0} g_{ \pm}$, тогда условие (6) запишется в виде

$$
\frac{1}{V} \int\left(g_{+}+g_{-}\right) d V=1 \text {. }
$$


Переходя в выражении (4) к безразмерным величинам, имеем

$$
\begin{aligned}
\widetilde{F}= & \frac{F}{K_{22} q_{0}^{2}}=\frac{1}{2}\left(\frac{d \varphi}{d \tilde{z}}-1\right)^{2}-\frac{1}{2} h^{2} \sin ^{2} \varphi \\
& -\xi h\left(g_{+}-g_{-}\right) \cos \varphi+\kappa\left(g_{+} \ln g_{+}+g_{-} \ln g_{-}\right) .
\end{aligned}
$$

Поскольку объемные доли $g_{+}$и $g$ - зависят только от $z$, условие (7) примет вид

$$
\frac{1}{\tilde{p}} \int_{0}^{\tilde{p}}\left(g_{+}+g_{-}\right) d \tilde{z}=1,
$$

где $\tilde{p} \equiv q_{0} p-$ шаг спирали в поле.

Полная свободная энергия

$$
\widetilde{\mathscr{F}} \equiv \frac{\mathscr{F}}{V K_{22} q_{0}^{2}}=\frac{1}{\tilde{p}} \int_{0}^{\tilde{p}} \widetilde{F} d \tilde{z}
$$

представляет собой функционал относительно $\varphi(z)$, $g_{+}(z)$ и $g_{-}(z)$, где $\widetilde{F}-$ безразмерная плотность полной свободной энергии (8). Равновесному состоянию суспензии отвечает минимум функционала (10).

Минимизируя (10) по $\varphi(z)$, получим

$$
\frac{d^{2} \varphi}{d \tilde{z}^{2}}=-\frac{1}{2} h^{2} \sin 2 \varphi+\xi\left(g_{+}-g_{-}\right) h \sin \varphi .
$$

Минимизация свободной энергии (10) по $g_{ \pm}(z)$ при условии постоянства числа частиц в образце (9) дает распределение концентрации частиц в двух магнитных подсистемах

$$
g_{ \pm}(\tilde{z})=Q \exp \left\{ \pm \frac{\xi h}{\kappa} \cos \varphi(\tilde{z})\right\} .
$$

Здесь нормировочный множитель $Q$ определяется из условия (9)

$$
Q^{-1}=\frac{2}{\tilde{p}} \int_{0}^{\tilde{p}} \cosh \left\{\frac{\xi h}{\kappa} \cos \varphi(\tilde{z})\right\} d \tilde{z} .
$$

Формула (12) описывает эффект сегрегации магнитной примеси, заключающийся в том, что частицы мигрируют вдоль оси спирали в те ее части, в которых минимальна их зеемановская энергия в поле $\mathbf{H}$.

Уравнение (11) имеет первый интеграл

$$
\frac{d \varphi}{d \tilde{z}}=\sqrt{A(\varphi)}
$$

где введено обозначение

$$
A(\varphi) \equiv C-h^{2} \sin ^{2} \varphi-2 \kappa\left(g_{+}+g_{-}\right) .
$$

Здесь $C$ - константа интегрирования. Мы выбрали знак плюс перед корнем в (14), полагая, что с ростом $z$ угол поворота директора и намагниченности растет (вращение против часовой стрелки).

В выражении (13) с помощью (14) можно перейти к интегрированию по углу $\varphi$, тогда получим

$$
Q^{-1}=\frac{2}{\tilde{p}} \int_{0}^{2 \pi} \cosh \left\{\frac{\xi h}{\kappa} \cos \varphi(\tilde{z})\right\} \frac{d \varphi}{\sqrt{A(\varphi)}} .
$$

Уравнение (14) позволяет определить неявную функцию $\varphi(\tilde{z}, h)$. Выбирая начало координат так, что $\varphi(0)=0$, находим

$$
\tilde{z}=\int_{0}^{\varphi(\tilde{z})} \frac{d \varphi}{\sqrt{A(\varphi)}}
$$

Шагу спирали $p$ соответствует изменение угла $\varphi$ на $2 \pi$, поэтому из уравнения (17) находим выражение для шага спирали в поле

$$
\tilde{p}=\int_{0}^{2 \pi} \frac{d \varphi}{\sqrt{A(\varphi)}}
$$

Константа интегрирования $C$ находится из условия минимума полной свободной энергии (10)

$$
\partial \widetilde{\mathscr{F}} / \partial C=0
$$

Вычисляя (10) с помощью (8), получим уравнение для $C$

$$
\int_{0}^{2 \pi} \sqrt{A(\varphi)} d \varphi=2 \pi
$$

Это позволяет с помощью (14) и (20) представить свободную энергию (10) в виде

$$
\widetilde{\mathscr{F}}=\frac{1-C}{2}+\kappa \ln (e Q) .
$$

Здесь $e$ - основание натуральных логарифмов.

Определим магнитные характеристики ФХ. Принимая во внимание (3) и (12), для приведенной намагниченности $\mathbf{M}=\mathscr{M} /\left(M_{s} f_{0}\right)$, где $\mathscr{M}=M_{s}\left(f_{+}-f_{-}\right) \mathbf{m}$, находим

$$
\mathbf{M}=2 Q \mathbf{m} \sinh \left\{\frac{\xi h}{\kappa} \cos \varphi\right\} .
$$

Это соотношение позволяет найти компоненты вектора приведенной намагниченности, усредненные по периоду спиральной структуры

$$
\langle\mathbf{M}\rangle=\frac{1}{\tilde{p}} \int_{0}^{2 \pi} \mathbf{M} d \tilde{z} .
$$


Переходя к интегрированию по $\varphi$ с помощью (14), получаем

$$
\begin{gathered}
\left\langle M_{x}\right\rangle=\left\langle M_{z}\right\rangle=0, \\
\left\langle M_{y}\right\rangle=\frac{2 Q}{\tilde{p}} \int_{0}^{2 \pi} \cos \varphi \sinh \left\{\frac{\xi h}{\kappa} \cos \varphi\right\} A^{-1 / 2}(\varphi) d \varphi .
\end{gathered}
$$

Таким образом, полная система уравнений (2), (3), (12), (16)-(18), (20) и (24) позволяет определить ориентационные и магнитные характеристики $Ф Х$.

\section{3. Поле перехода в ферронематическую фазу}

Построенная система уравнений ориентационного и магнитного состояний ФХ позволяет изучить влияние магнитного поля, приложенного перпендикулярно оси спирали, на ориентационную структуру и магнитные свойства ФХ. Как уже отмечалось, такое поле вызывает раскручивание спиральной структуры ФХ при достижении напряженностью поля критического значения $h_{c}$, которое определяется из условия равенства свободной энергии ФХ (21) и свободной энергии раскрученного полем ферронематика $(\Phi \mathrm{H})$

$$
\begin{aligned}
\widetilde{F}_{0}=\frac{1}{2} & -\frac{1}{2} h^{2} \sin ^{2} \varphi_{c}-\xi h\left(g_{0+}-g_{0-}\right) \cos \varphi_{c} \\
& +\kappa\left(g_{0+} \ln g_{0+}+g_{0-} \ln g_{0-}\right),
\end{aligned}
$$

где $\varphi_{c}-$ не зависящий от координат угол ориентации директора в ФН-фазе и введена концентрация магнитных частиц в ферронематической фазе

$$
\begin{gathered}
g_{0 \pm}=Q_{c} \exp \left\{ \pm \frac{\xi h}{\kappa} \cos \varphi_{c}\right\} \\
Q_{c}^{-1}=2 \cosh \left\{\frac{\xi h}{\kappa} \cos \varphi_{c}\right\} .
\end{gathered}
$$

Значение угла $\varphi_{c}$ определяется условиями минимума $\partial \widetilde{F}_{0} / \partial \varphi_{c}=0$ свободной энергии $(25)$. Одно из решений этого уравнения $\left(\varphi_{c}=0\right)$ отвечает раскрученной полем ФН-фазе, в которой, согласно формулам (2) и (3), имеем $\mathbf{m}=(0,1,0), \mathbf{n}=(1,0,0)$, т. е. $\mathbf{n} \perp \mathbf{m} \| \mathbf{H}$. В такой

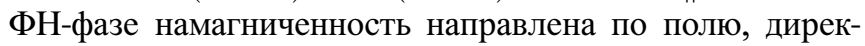
тор перпендикулярен полю. Назовем такую фазу $\Phi H_{\|}$. В этом случае переход из $\Phi$ Х-фазы в фазу $\Phi \mathrm{H}_{\|}$обеспечивается дипольным (ферромагнитным) механизмом. Фаза $\Phi \mathrm{H}_{\|}$устойчива, если

$$
\frac{\xi}{h} \tanh \left\{\frac{\xi h}{\kappa}\right\} \geq 1 .
$$

Другое решение $\varphi_{c}=\pi / 2$ уравнения $\partial \widetilde{F}_{0} / \partial \varphi_{c}=0$ отвечает $\mathbf{m}=(1,0,0), \mathbf{n}=(0,1,0)$ (т.е. в раскрученной полем ФН-фазе $\mathbf{m} \perp \mathbf{n} \| \mathbf{H})$ и является термодинамически устойчивым при $\xi^{2} \leq \kappa$. Назовем такую фазу $\Phi H_{\perp}$, так как в ней директор направлен по полю, а намагниченность перпендикулярна полю. Раскручивание спиральной структуры ФХ в этом случае обеспечивается квадрупольным (диамагнитным) механизмом.

В точке перехода $\Phi \mathrm{X}-\Phi Н$, т. е. при $h=h_{c}$, свободные энергии (21) и (25) должны быть одинаковыми. Это позволяет найти значение константы $C$ в точке перехода

$$
C_{c}=2 \kappa+h_{c}^{2} \sin ^{2} \varphi_{c}
$$

Подставляя теперь $C_{c}$ в уравнение (20), находим уравнение для критического поля $h_{c}$ перехода из $\Phi Х$-фазы в ФН-фазу

$$
\begin{aligned}
\int_{0}^{2 \pi} d \varphi \sqrt{\begin{array}{c}
2 \kappa\left[1-\cosh \left(\frac{\xi h_{c}}{\kappa} \cos \varphi\right)\left\{\cosh \left(\frac{\xi h_{c}}{\kappa} \cos \varphi_{c}\right)\right\}^{-1}\right] \\
+h_{c}^{2}\left(\sin ^{2} \varphi_{c}-\sin ^{2} \varphi\right)
\end{array}} \\
=2 \pi
\end{aligned}
$$

где $\varphi_{c}$ - одно из указанных выше решений: $\varphi_{c}=0$ или $\varphi_{c}=\pi / 2$.

Заметим, что в точке перехода в ФН-фазу (т.е. при $\left.h=h_{c}\right)$

$$
\begin{gathered}
g_{0 \pm}=Q_{c} \exp \left\{ \pm\left(\xi h_{c} \cos \varphi_{c}\right) / \kappa\right\}, \\
Q_{c}=(1 / 2) \cosh ^{-1}\left\{\xi h_{c} \cos \varphi_{c} / \kappa\right\}, \\
\left\langle M_{y}\right\rangle_{c}=\tanh \left\{\frac{\xi h_{c}}{\kappa} \cos \varphi_{c}\right\} .
\end{gathered}
$$

Согласно формуле $(31)$, в фазе $\Phi H_{\|}$, в которой $\varphi_{c}=0$, находим $\left\langle M_{y}\right\rangle_{c} \neq 0$, т. е. $Ф Н$ не является компенсированным $\left(g_{0+}-g_{0-} \neq 0\right)$, и магнитные подсистемы упорядочены по типу ферримагнетика. Отсюда же видно, что в фазе $\Phi \mathrm{H}_{\perp}$, в которой $\varphi_{c}=\pi / 2$, находим $g_{0+}=g_{0-} \neq 0$ и $\left\langle M_{y}\right\rangle_{c}=0$, т. е. такая $\Phi$ Н-фаза антиферромагнитна.

Представим уравнения для $C, Q$ и $\left\langle M_{y}\right\rangle$ в удобном для выделения асимптотических зависимостей виде, чтобы определить поведение шага спирали и намагниченности в подкритической области (дипольный режим). Для этого перепишем уравнение для шага (18)

$$
C=2 \kappa+h^{2} \sin ^{2} \varphi_{c}+\frac{2 \pi}{\tilde{p}}-\frac{h^{2}}{\tilde{p}} J_{1},
$$

где

$$
J_{1}=\int_{0}^{2 \pi} \cos ^{2} \varphi \frac{d \varphi}{\sqrt{A(\varphi)}} .
$$

В уравнении (16) для $Q$ выделяем асимптотическое значение

$$
Q=\frac{(1 / 2) \cosh ^{-1}\left\{\xi h \cos \varphi_{c} / \kappa\right\}}{1-J_{2} / \tilde{p}}
$$

где

$$
J_{2}=\int_{0}^{2 \pi}\left(1-\exp \left\{2 \frac{\xi h}{\kappa} \cos ^{2}\left(\frac{\varphi}{2}\right)\right\}\right) \frac{d \varphi}{\sqrt{A(\varphi)}}
$$


Из уравнения (24) получаем

$$
\left\langle M_{y}\right\rangle=\tanh \left\{\frac{\xi h \cos \varphi_{c}}{\kappa}\right\}\left[\frac{1}{1-J_{2} / \tilde{p}}-\frac{2 J_{3}}{\tilde{p}}\right],
$$

где

$$
J_{3}=\int_{0}^{2 \pi} \sin ^{2}\left(\frac{\varphi}{2}\right) \exp \left\{2 \frac{\xi h}{\kappa} \cos ^{2}\left(\frac{\varphi}{2}\right)\right\} \frac{d \varphi}{\sqrt{A(\varphi)}} .
$$

Здесь интегралы $J_{1}, J_{2}$ и $J_{3}$ остаются конечными при $h \rightarrow h_{c}$.

\section{4. Шаг спирали и намагниченность}

В отсутствие поля ФХ-фаза не возмущена, но с ростом напряженности поля спиральная структура начинает деформироваться, шаг спирали растет и обращается в бесконечность (переход $Ф \mathrm{X}-\Phi Н)$ при $h=h_{c}$. На рис. 1 показаны зависимости критического поля $h_{c}$ перехода в ферронематическую фазу от параметра $\xi$ при различных значениях параметра сегрегации $\kappa$, построенные путем численного решения уравнения (29). Вертикальные штриховые линии на этом рисунке отвечают значению $\xi^{2}=\kappa$.

Как видно из рис. 1, характер решений уравнения (29) в случае компенсированного ФХ зависит от значения параметра $\xi^{2} / \kappa$. При $\xi<\xi_{*}=\sqrt{\kappa}$ преобладающим является типичный для жидких кристаллов квадрупольный механизм влияния поля, и раскручивание спиральной структуры ФХ происходит в полях, бо́льших $\pi / 2$, т.е. поля перехода в чистом ХЖК. Кривые 1 и 2 на рис. 1 отвечают полю перехода $\Phi \mathrm{X}-\Phi Н$. Область, лежащая выше этих кривых $h_{c}(\xi)$, отвечает антиферромагнитной фазе $\Phi H_{\perp}$ с компенсированной магнитной примесью.

При $\xi>\xi_{*}$ зависимость $h_{c}(\xi)$ двузначна: заданному значению $\xi$ отвечают два значения $h_{c}$. Область, ограниченная кривыми $1^{\prime}$ и $2^{\prime}$ на рис. 1 , отвечает фазе $\Phi \mathrm{H}_{\|}$, под кривыми и над ними - ФХ-фазе. Как видно из рис. 1 , для заданных $\kappa$ и $\xi>\xi_{*}$ меняется последовательность переходов, происходящих в ФХ под действием магнитного поля. Для заданного $\xi$ значение $h_{c}$ на нижней ветви кривых $1^{\prime}$ и $2^{\prime}$ фазового равновесия отвечает переходу в фазу $\Phi \mathrm{H}_{\|}$, которая остается устойчивой до достижения полем значения $h_{c}$, соответствующего верхней ветви этих кривых, где преобладающим является квадрупольный механизм. Дальнейшее увеличение поля приводит к закручиванию холестерической спирали и, следовательно, появлению возвратной ФХ-фазы. Таким образом, в данной области значений параметра $\xi$ при увеличении поля суспензия претерпевает последовательность фазовых переходов феррохолестерикферронематик-феррохолестерик. Появление возвратной феррохолестерической фазы является результатом сегрегационных процессов и конкурирующего влияния дипольного и квадрупольного ориентационных механиз-

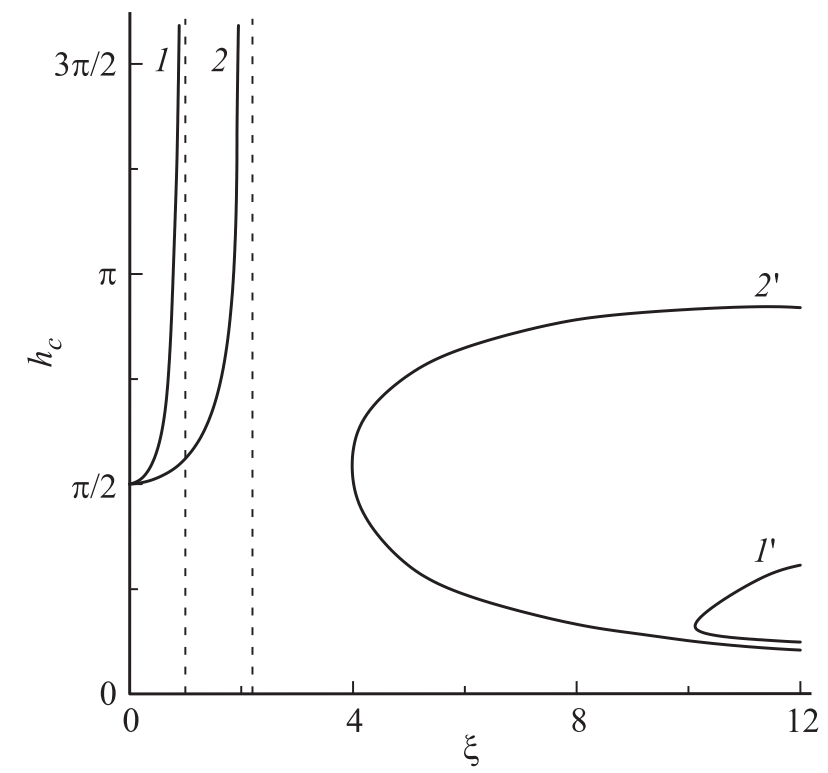

Рис. 1. Зависимость $h_{c}(\xi)$ при $\kappa=1$ (кривые 1 и $l^{\prime}$ ) и 5 (кривые 2, и $2^{\prime}$ ).

мов, которые стремятся раскрутить спиральную структуру в противоположных направлениях. Заметим, что поле перехода в нематическую фазу в чистом ХЖК равно в безразмерных единицах $\pi / 2$. Поэтому, как видно из рис. 1 , присутствие магнитной примеси при $\xi>\xi_{*}$ значительно понижает поле раскручивания спиральной структуры ФХ по сравнению с величиной для чистого ХЖК. Это следует из уравнения (29): при $h_{c} \ll 1$ уравнение дает $h_{c} \approx(\pi / 2) /\left[\xi^{2} / \kappa-1\right]^{-1 / 2}$, отсюда $h_{c} \rightarrow 0$ при $\xi \gg 1$ (дипольный режим).

Как видно из рис. 1, имеется интервал значений параметра $\xi$, такой, что при $\xi_{*}<\xi<\xi_{0}$, где $\xi_{0}-$ значение параметра $\xi$, отвечающее вершине кривой $1^{\prime}$

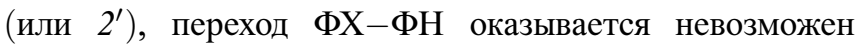
вследствие конкуренции дипольного и квадрупольного ориентационного механизмов. Этот интервал значений параметра $\xi$ растет по мере уменьшения параметра $\kappa$, т. е. при увеличении интенсивности сегрегации (ср. кривые $1,1^{\prime}$ и $2,2^{\prime}$ ).

На рис. 2 показаны зависимости шага спирали и намагниченности компенсированного ФХ от напряженности поля для $\xi=1<\xi_{*}=\sqrt{\kappa}$, что отвечает кривым 1 и 2 на рис. 1, при двух значениях параметра сегрегации $\kappa$. Видно, что шаг спирали с ростом поля увеличивается быстрее при малых $\kappa$, т.е. при существенной сегрегации. Напомним, что в отсутствие поля ФХ находился в компенсированном состоянии, в котором $f_{+}=f_{-}=f_{0} / 2$, поэтому намагниченность $\mathscr{M}=M_{s}\left(f_{+}-f_{-}\right) \mathbf{m}=0$, несмотря на наличие геликоидальной закрученности (3). Из рис. $2, b$ видно, что в точке перехода $h=h_{c}$ в фазу $\Phi \mathrm{H}_{\perp}$ суспензия вновь находится в компенсированном состоянии (при $h=h_{c}$ намагниченность равна нулю согласно формуле (31), 
в которой теперь $\varphi_{c}=\pi / 2$, т.е. магнитные моменты частиц упорядочены по типу антиферромагнетика).

На рис. 3 показаны зависимости намагниченности и шага спирали $\Phi \mathrm{X}$ от напряженности поля для $\xi=5>\xi_{*}$ (см. кривую $2^{\prime}$ на рис. 1). В этом случае, как видно из рис. 1 , при заданном $\xi=5$ и $\kappa=5$ имеется два критических значения поля $h_{c 1}=1.017$ и $h_{c 2}=2.437$.

Из рис. 3, $a$ видно, что с ростом поля шаг спирали растет и обращается в бесконечность при $h=h_{c 1}$. Здесь, как отмечалось выше, спиральная структура $\Phi Х$ раскручивается вследствие дипольного (ферромагнитного) механизма. Область $h_{c 1} \leq h \leq h_{c 2}$ соответствует фазе $\Phi \mathrm{H}_{\|}$. В полях $h>h_{c 2}$ суспензия вновь приобретает спиральную структуру (этот диапазон значений напряженности
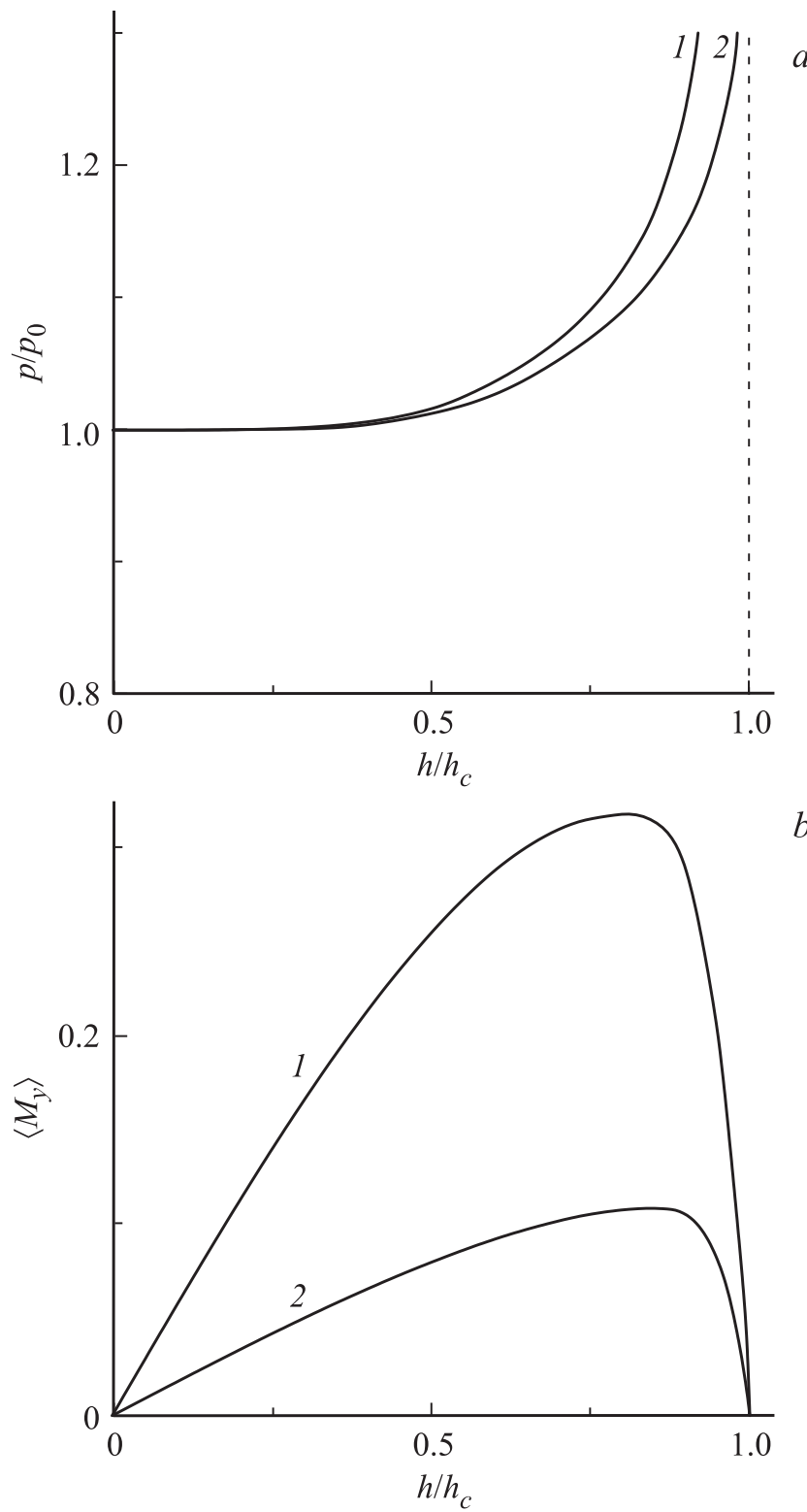

Рис. 2. Зависимость шага спирали $p(a)$ и средней намагниченности $\left\langle M_{y}\right\rangle(b)$ от напряженности поля для $\xi=1$. Кривая $1-\kappa=2\left(h_{c}=2.315\right)$, кривая $2-\kappa=5\left(h_{c}=1.762\right)$.
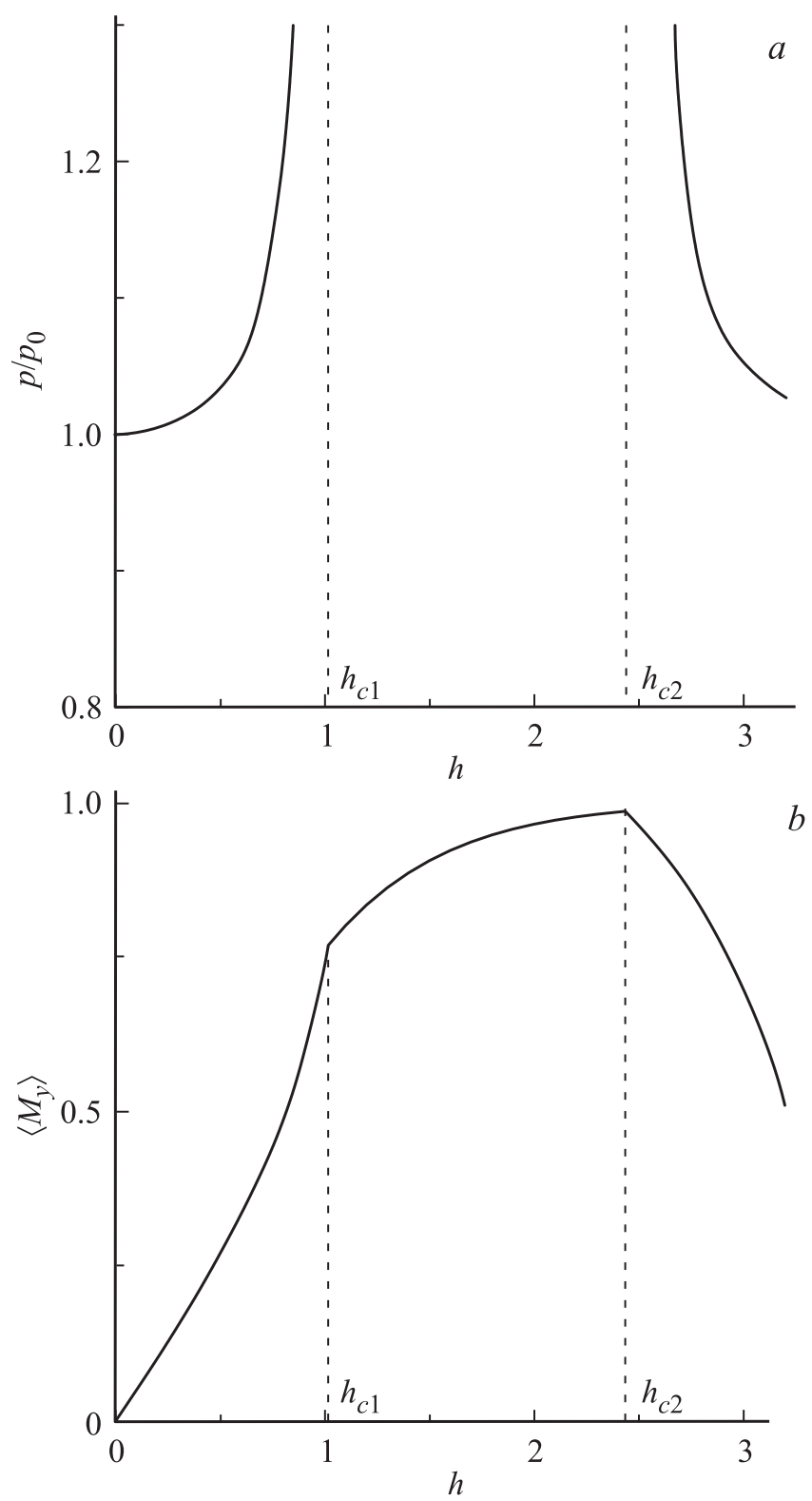

Рис. 3. Зависимость шага спирали $p(a)$ и средней намагниченности $\left\langle M_{y}\right\rangle(b)$ от напряженности поля для $\xi=\kappa=5$ $\left(h_{c 1}=1.017\right.$ и $\left\langle M_{y}\right\rangle_{c 1}=0.768, h_{c 2}=2.437$ и $\left.\left\langle M_{y}\right\rangle_{c 2}=0.985\right)$.

поля отвечает возвратной ФХ-фазе). Это связано с тем, что в достаточно сильных полях квадрупольный (диамагнитный) механизм становится столь же существенным, что и дипольный. Дальнейшее поведение шага спирали обусловлено именно им.

На рис. 3,b представлена зависимость средней намагниченности компенсированного $\Phi \mathrm{X}$ от напряженности магнитного поля. В слабых полях $\left(h \ll h_{c}\right)$ среднюю намагниченность (24) можно представить в виде $\left\langle M_{y}\right\rangle \approx \xi h /(2 \kappa)$, что соответствует начальному участку построенных кривых. Отсюда можно найти начальную восприимчивость $\chi \approx \chi_{a} \xi^{2} /(2 \kappa)$. Намагниченность при достижении критического поля $h=h_{c 1}$ находится из 
выражения (31). При $h_{c 1} \leq h \leq h_{c 2}$ суспензия находится в фазе $\Phi \mathrm{H}_{\|}$, в которой $\varphi_{c}=0$, поэтому, согласно (31), имеем $\left\langle M_{y}\right\rangle=\tanh \{\xi h / \kappa\}$, и намагниченность в направлении поля растет, стремясь к насыщению, но не достигая его $\left(\left\langle M_{y}\right\rangle<1\right)$, так как при $h=h_{c 2}$ разность $\left(g_{+}-g_{-}\right) \ll 1$ и магнитная структура фазы $\Phi \mathrm{H}_{\|}$отвечает слабому ферримагнетику.

\section{Заключение}

В работе изучен фазовый переход компенсированного ФХ с гомеотропным сцеплением магнитных частиц с

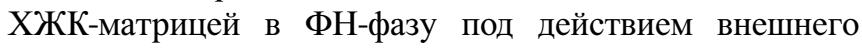
магнитного поля, перпендикулярного оси спиральной структуры. Показана возможность существования двух различных по магнитной структуре ферронематических

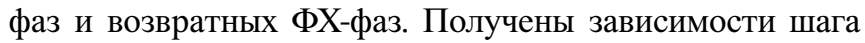
спирали и средней намагниченности от магнитного поля. Показано, что наличие магнитной примеси значительно уменьшает величину поля перехода в ФН-фазу в дипольном режиме по сравнению с величиной для чистого ХЖК.

\section{Список литературы}

[1] L.M. Blinov. Structure and properties of liquid crystals. Springer, Dordrecht (2011). 439 p.

[2] F. Brochard, P.G. de Gennes. J. de Phys. (France) 31, 691 (1970).

[3] S.-H. Chen, S.H. Chiang. Mol. Cryst. Liq. Cryst. 144, 359 (1987).

[4] O. Kurochkin, O. Buchnev, A. Iljin, S.K. Park, S.B. Kwon, O. Grabar, Yu. Reznikov. J. Opt. A 11, 024003 (2009).

[5] W. Hu, H.Y. Zhao, L.K. Shan, L. Song, H. Cao, Z. Yang, Z.H. Cheng, C.Z. Yan, S.J. Li, H.A. Yang, L. Guo. Liq. Cryst. 37, 563 (2010).

[6] H. Ayeb, J. Grand, H. Sellame, S. Truong, J. Aubard, N. Felidj, A. Mlayah, E. Lacaze. J. Mater. Chem. 22, 7856 (2012).

[7] M. Infusino, A. De Luca, F. Ciuchi, A. Ionescu, N. Scaramuzza, G. Strangi. Mol. Cryst. Liq. Cryst. 572, 59 (2013).

[8] M. Infusino, A. De Luca, F. Ciuchi, A. Ionescu, N. Scaramuzza, G. Strangi. J. Mater. Sci. 49, 1805 (2014).

[9] J.S. Pendery, O. Merchiers, D. Coursault, J. Grand, H. Ayeb, R. Greget, B. Donnio, J.-L. Gallani, C. Rosenblatt, N. Félidj, Y. Borensztein, E. Lacaze. Soft Matter 9, 9366 (2013).

[10] B. Matt, K.M. Pondman, S.J. Asshoff, B. ten Haken, B. Fleury, N. Katsonis. Angew. Chem. Int. Ed. 53, 12446 (2014).

[11] B. Senyuk, M.C.M. Varney, J.A. Lopez, S. Wang, N. Wuc, I.I. Smalyukh. Soft Matter 10, 6014 (2014).

[12] B.T.P. Madhav, P. Pardhasaradhi, R.K.N.R. Manepalli, V.G.K.M. Pisipati. Liq. Cryst. 42, 989 (2015).

[13] Q. Zhang, P.J. Ackerman, Q. Liu, I.I. Smalyukh. Phys. Rev. Lett. 115, 097802 (2015).

[14] A.N. Zakhlevnykh, P.A. Sosnin. J. Magn. Magn. Mater. 146, 103 (1995).

[15] E. Petrescu, C. Motoc. J. Magn. Magn. Mater. 234, 142 (2001).

[16] A. Zakhlevnykh, V. Shavkunov. Mol. Cryst. Liq. Cryst. Sci. Technol. A 330, 593 (1999).
[17] A.N. Zakhlevnykh, V.S. Shavkunov. J. Magn. Magn. Mater. 210, 279 (2000).

[18] V.S. Shavkunov, A.N. Zakhlevnykh. Mol. Cryst. Liq. Cryst. Sci. Technol. A 367, 175 (2001).

[19] E. Petrescu, E.-R. Bena. J. Magn. Magn. Mater. 320, 299 (2008).

[20] H.R. Brand, A. Fink, H. Pleiner. Eur. Phys. J. E 38, 65 (2015).

[21] А.Н. Захлевных, К.В. Кузнецова. ФТТ 58, 2274 (2016).

[22] А.Н. Захлевных, Д.А. Петров. ЖТФ 82, 9, 28 (2012).

[23] A.N. Zakhlevnykh, D.A. Petrov. J. Magn. Magn. Mater. 401, 188 (2016) 